\title{
Predição do campo térmico bidimensional de estruturas em situação de incêndio via elementos finitos
}

\author{
Natan Sian das Neves ${ }^{1}$ \\ Programa de Engenharia Civil, COPPE/UFRJ, Rio de Janeiro, RJ \\ Rodrigo Silveira Camargo ${ }^{2}$ \\ Programa de Pós-Graduação em Engenharia Civil, UFES, Vitóra, ES \\ Macksuel Soares de Azevedo ${ }^{3}$ \\ Programa de Pós-Graduação em Engenharia Civil, UFES, Vitóra, ES
}

\begin{abstract}
Resumo. A área de estruturas em condição de incêndio direciona um vasto volume de pesquisa cientifica aplicada na engenharia. Um elemento estrutural quando sujeito a elevadas temperaturas apresenta degradação das propriedades físicas dos materiais conjunto com a perda de sua capacidade portante. Neste cenário, o conhecimento da distribuição de temperatura na seção transversal torna-se importante para análises e dimensões mínimas em projetos. O presente trabalho apresenta uma ferramenta computacional destinada à análise térmica não linear bidimensional de elementos estruturais em condição de incêndio. O programa tem como base os procedimentos de elementos finitos de Galerkin, possibilitando realizar análises em geometrias não estruturadas com propriedades termofísicas variando com temperatura e sujeitos a qualquer curva de incêndio em função do tempo. A experimentação numérica é direcionada por meio da análise de estruturas de concreto e mistas de aço e concreto, sendo que os resultados numéricos são comparados com procedimentos normativos, simulações computacionais ou dados experimentais disponíveis na literatura. Em síntese, os resultados obtidos mostram-se satisfatórios, indicando o bom desempenho do programa diante os problemas propostos.
\end{abstract}

Palavras-chave. Elementos Finitos, Incêndio, Análise Térmica, Concreto-Aço.

\section{Introdução}

No decorrer da linha histórica, ocorrências e prejuízos materiais devido aos incêndios são inevitáveis. O desenvolvimento de pesquisas cientificas correlacionadas aos avanços tecnológicos corroboram para aprimoramentos dos procedimentos e métodos relacionados ao combate e segurança contra incêndio. Sendo assim, foram elaborados inúmeras documentações sobre incêndio, por exemplo, regulamentações, instruções técnicas e normas. Dentre elas, cita-se as normas brasileiras, como a NBR 14323:2013 [1] e NBR 15200:2012 [2], e as normas internacionais, EN 1992-1-2:2004 [4] e EN 1993-1-2:2005 [4].

No contexto de projetos de estruturas de concreto e/ou aço em situação de incêndio, usualmente aplicam-se os procedimentos simplificados de cálculo - que possibilitam realizar análises em configurações simples e com certas restrições de uso. Todavia, existem problemas práticos que não podem ser solucionados com os métodos simplificadas normativos, demandando a aplicação de

\footnotetext{
${ }^{1}$ natan.neves@coppe.ufrj.br

${ }^{2}$ rodrigo_camargo2000@yahoo.com

${ }^{3}$ macksuel.azevedo@gmail.com
} 
métodos avançados de cálculo, como os elementos estruturais com seções transversais não convencionais, os elementos compostos com materiais diferentes, o comportamento mecânico avançado dos elementos em altas temperaturas ou estruturas de grande porte. Sendo assim, independente da análise realizada, o conhecimento do campo de temperatura no interior das estruturas é uma etapa inevitável e importante em projetos de estruturas em situação de incêndio.

Os modelos numéricos, por conta da versatilidade, agilidade de análise e desempenho computacional, ganharam espaço em projetos acadêmicos e aplicações industriais. Neste cenário, o presente trabalho visa apresenta um módulo computacional específico para análise térmica de estruturas em situação de incêndio, denotado como NASEN/TA-FIRE (Numerical Analysis System for Engineering/Thermal Analysis - Fire) [9]. Atualmente, no Brasil, pode-se mencionar linhas de pesquisa semelhantes, como apresentado em Pierin et al. [10] e Pires et al. [11], que desenvolveram programas computacionais para análise térmica em incêndio, ou em Landesmann [7] que desenvolveu em um dos tópicos da pesquisa, um modelo térmico unidimensional para incêndio.

\section{Formulação numérica}

A formulação de elementos finitos para a análise térmica baseia-se na técnica de resíduos ponderados - que tem como objetivo realizar uma minimização de resíduo. Sendo assim, inicialmente, é necessário o conhecimento prévio do modelo matemático do fenômeno físico, ou seja, com base no princípio da conservação de energia e considerando que o fluxo de calor é descrito pela lei de Fourier, a equação de governo do problema de condução de calor em um corpo sólido é expressa pela equação (1) [3].

$$
\nabla^{T} \mathbf{D}(T) \nabla T+\dot{Q}=\rho(T) c(T) \frac{\partial T}{\partial t}
$$

onde $T$ é a temperatura, $\dot{Q}$ é a geração de energia interna, $\rho$ é a massa específica do material, $c$ é o calor específico e $\mathbf{D}$ é conhecida como matriz de condutividade térmica do material. Para o caso de um material isotrópico bidimensional, vale a equação (2).

$$
\mathbf{D}=\left[\begin{array}{ll}
k & 0 \\
0 & k
\end{array}\right]=k(T) \mathbf{I}
$$

Em particular ao comportamento térmico de estruturas em condição de incêndio, as propriedades dos materiais variam com o aumento de temperatura, ou seja, o problema torna-se não linear. As expressões matemáticas das propriedades termofísicas do aço e do concreto seguem, respectivamente, as descrições do EN 1993-1-2:2005 [5] e EN 1992-1-2:2004 [4].

As condições de contorno de um elemento exposto ao incêndio são esquematizadas na Figura 1. A condição do tipo essencial é descrita pela consideração de temperatura prescrita no contorno, enquanto, a condição do tipo natural é definida pela imposição do fluxo de calor combinado de convecção-radiação, na forma linearizada, conforme expresso a seguir:

$$
q_{n}=\alpha_{c}\left(T_{s}-T_{b}\right)+\alpha_{r}\left(T_{s}-T_{b}\right), \quad \alpha_{r}=\epsilon \sigma\left(T_{s}-T_{b}\right)\left(T_{s}^{2}+T_{b}^{2}\right)
$$

onde $T_{s}=T$ é a temperatura do sólido, $\sigma$ é a constante de Stefan-Boltzmann $\left(=5,6697 \times 10^{-8}\right.$ $\left.\mathrm{W} / \mathrm{m}^{2} \mathrm{~K}^{4}\right), \epsilon$ é a emissividade, $\alpha_{c}$ e $\alpha_{r}$ são, respectivamente, o coeficiente de convecção e de radiação. Além disso, a temperatura dos gases quentes $T_{b}$ é descrita pela norma ISO 834:1999 [6], conforme expresso na equação (4). 


$$
T_{b}=T_{a m b}+345 \log (8 t+1)
$$

onde $t$ é o tempo de exposição, em minutos, e $T_{a m b}$ é a temperatura ambiente em graus Celsius. A equação (4) é usualmente denominada como curva de incêndio-padrão ISO 834:1999 [6] e nota-se que tal é dada por uma relação logarítmica crescente.

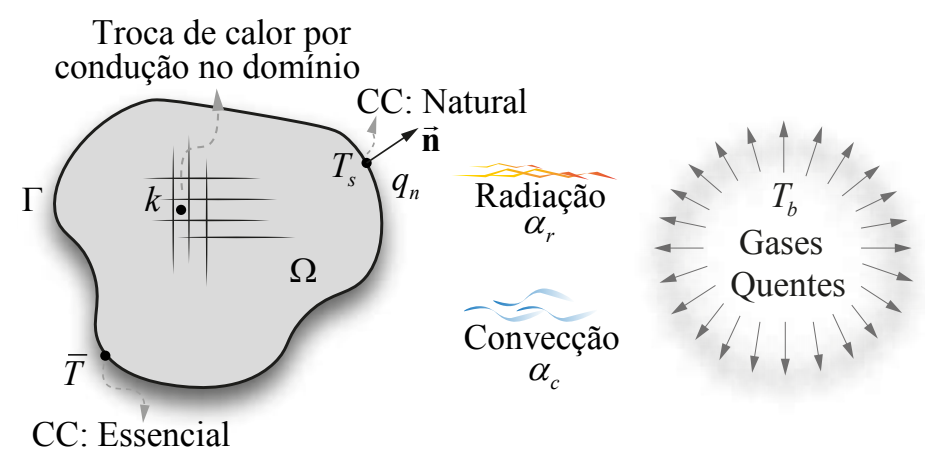

Figura 1: Condições de contorno de natureza térmica no domínio sólido bidimensional

Após a exposição das principais equações do comportamento térmico de estruturas bidimensionais, a formulação do método de elementos finitos para problemas não lineares de difusão de calor é definida escrevendo a equação (1) como uma sentença integral conjunta com uma função auxiliar (ou peso) $w$. Aplicando a regra da cadeia e com algumas manipulações algébricas [12], é possível chegar na equação (5).

$$
\int_{\Omega} \nabla^{T} w(\mathbf{D} \nabla T) d \Omega+\int_{\Omega} \rho c \frac{\partial T}{\partial t} w d \Omega=\int_{\Omega} \dot{Q} w d \Omega-\int_{\Gamma} q_{n} w d \Gamma
$$

A equação (4) é conhecida como formulação variacional fraca e não apresenta nenhuma aproximação numérica, sendo que todo o procedimento é exato e realizado em torno de um domínio contínuo $\Omega$. Escrevendo a função resposta aproximada como uma combinação de coeficientes e funções de interpolação $N$, conforme dado pela equação (6)

$$
T \approx \sum_{i=1}^{N} N_{i} T_{i}=\mathbf{N}^{\mathrm{T}} \tilde{T} \quad w \approx \sum_{j=1}^{N} N_{j} w_{j}=\mathbf{N}^{\mathrm{T}} \tilde{w}
$$

e substituindo as aproximações na equação (5), com algumas manipulações matemáticas, chega-se na forma matricial compacta do sistema, como é representado na equação (7).

$$
\mathbf{C} \frac{\partial \tilde{T}}{\partial t}+\mathbf{K} \tilde{T}=\mathbf{F}
$$

onde $\mathbf{C}$ é a matriz de capacidade térmica, $\mathbf{K}$ é a matriz de condutividade térmica total e $\mathbf{F}$ é o vetor térmico ${ }^{4}$, dadas por:

$$
\mathbf{K}=\mathbf{K}^{e}+\mathbf{H}^{e}+\mathbf{R}^{e} \quad \text { e } \quad \mathbf{F}=\mathbf{F}_{\dot{Q}}+\mathbf{F}_{\bar{q}}+\mathbf{F}_{h}+\mathbf{F}_{r}
$$

\footnotetext{
${ }^{4}$ No contexto da análise dinâmica de estruturas, as matrizes são, respectivamente, denominadas de matriz de amortecimento, matriz de rigidez e vetor de força
} 
As matrizes $\mathbf{H}^{e}$ e $\mathbf{F}_{h}$ são responsáveis pela contribuição da ação do fluxo de convecção do contorno, e as matrizes $\mathbf{R}^{e}$ e $\mathbf{F}_{r}$ representam a parcela do fluxo de radiação. Em condição de incêndio, os vetores de geração de calor e de fluxo prescrito no contorno são desprezados $\left(\mathbf{F}_{\dot{Q}}=\right.$ $\left.\mathbf{F}_{\bar{q}}=0\right)$. A equação (7) indica uma natureza transiente, isto é, o campo de temperatura varia com o tempo. Desta maneira, usando o método de diferenças finitas para aproximação do operador derivada temporal, é possível escrever a temperatura no instante $n+1$ [12], como apresentado na equação (9).

$$
\left(\frac{\mathbf{C}}{\Delta t}+\theta \mathbf{K}\right) \tilde{T}_{n+1}=\left(\frac{\mathbf{C}}{\Delta t}-(1-\theta) \mathbf{K}\right) \tilde{T}_{n}+(1-\theta) \mathbf{F}_{n}+\theta \mathbf{F}_{n+1}
$$

Em que $\Delta t$ é o intervalo de tempo e o parâmetro $\theta$ que varia de 0 a 1 , representando o esquema de integração no tempo. No presente trabalho adota-se 2/3, que caracteriza o esquema de Galerkin, sendo esse incondicionalmente estável [9, 12].

\section{Experimentação numérica}

A experimentação é constituída por três casos testes, conforme ilustra a Figura 2. Em todas as simulações, as estruturas são submetidas à curva padrão de incêndio ISO 834:1999 [6] e a temperatura ambiente é adotada como sendo igual a $20^{\circ} \mathrm{C}$. Desta forma, o primeiro caso é uma laje retangular de concreto de altura igual a $200 \mathrm{~mm}$ exposta ao fogo pela face inferior, tendo as laterais isoladas termicamente, como mostra a Figura 2a. A emissividade é adotada igual a 0,7 e o coeficiente de convecção para as faces expostas ao incêndio é igual a $25 \mathrm{~W} / \mathrm{m}^{2 \circ} \mathrm{C}$. Esses parâmetros térmicos são utilizados no cálculo do fluxo de calor combinado de convecção-radiação, conforme apresentado na equação (3) [4-5].

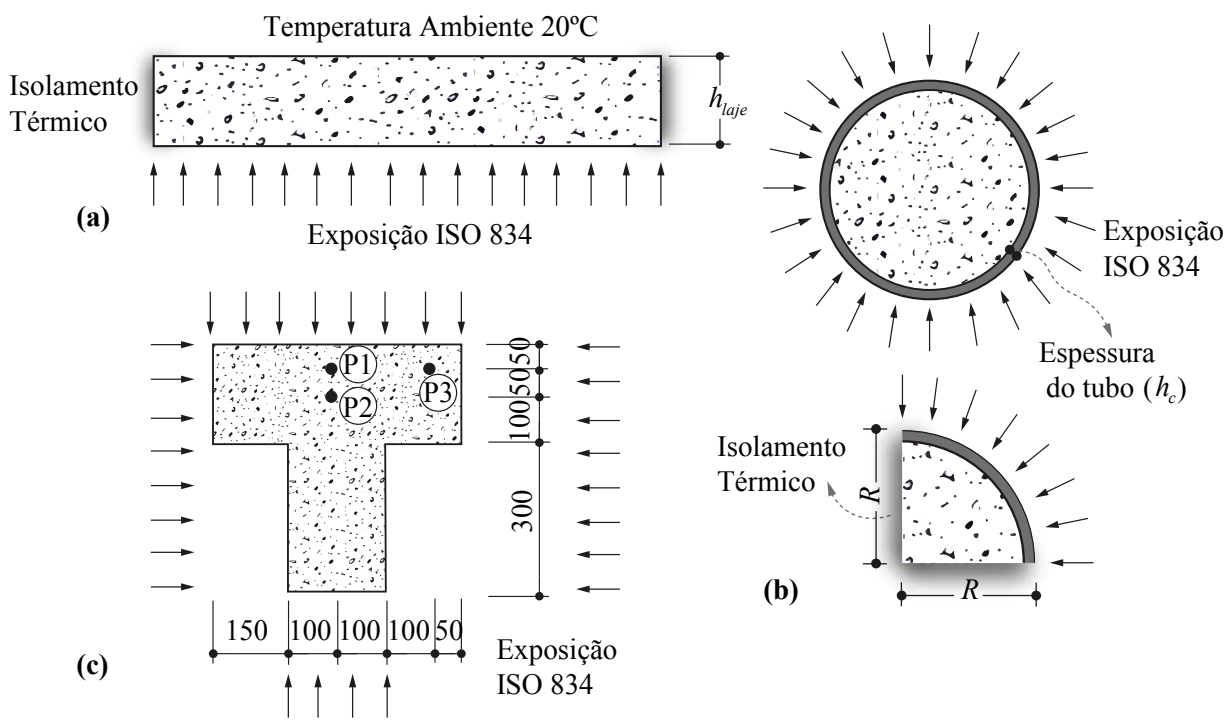

Figura 2: Estudo de caso: (a) laje de concreto de espessura constante, (b) tubo circular preenchido com concreto e (c) pilar em forma de $\mathrm{T}$ de concreto.

A laje retangular de concreto com as devidas configurações estudadas torna-se um problema unidimensional, pelo fato da imposição de fluxo de calor nulo nas laterais. Os resultados numéricos 
obtidos com a simulação computacional são comparados com os dados obtidos no EN 1992-1-2:2004 [4]. A Figura 3 apresenta os perfis de temperatura para 30, 60, 90 e 120 min de exposição ao incêndio. Observa-se que os resultados numéricos apresentam um comportamento semelhante com os dados normativos, indicando um bom desempenho do programa. Pelo fato da laje estar exposta ao fogo na face inferior, as temperaturas diminuem com o aumento da altura da laje.

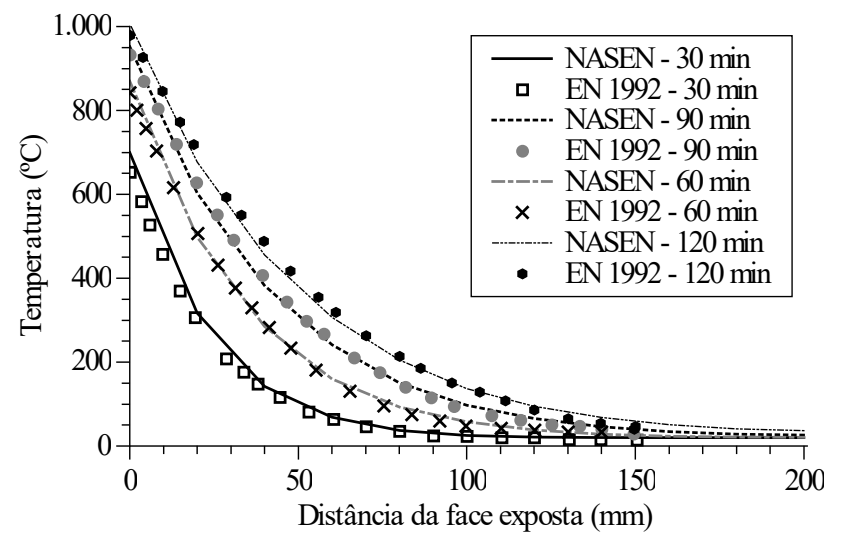

\section{Malha de Elementos Finitos}

Elemento Triangular

$\#$ nox $=305 \quad \#$ elem $=608$

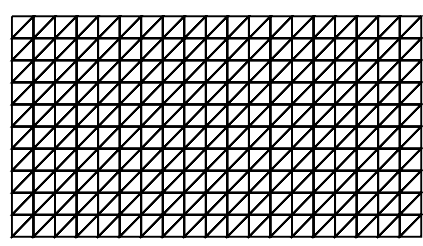

Figura 3: Perfil de temperatura da laje de concreto com espessura $200 \mathrm{~mm}$ e malha numérica.

O segundo caso é um perfil de aço tubular preenchido de concreto, conforme apresenta a Figura 2b. Os valores de emissividade e de coeficiente de convecção são iguais ao caso anterior. Considerase o diâmetro do tubo circular igual a $500 \mathrm{~mm}$ e a espessura do tubo igual a $20 \mathrm{~mm}$. A avaliação dos resultados do presente trabalho é realizado com base nos resultados numéricos obtidos em Yin et al. [14].

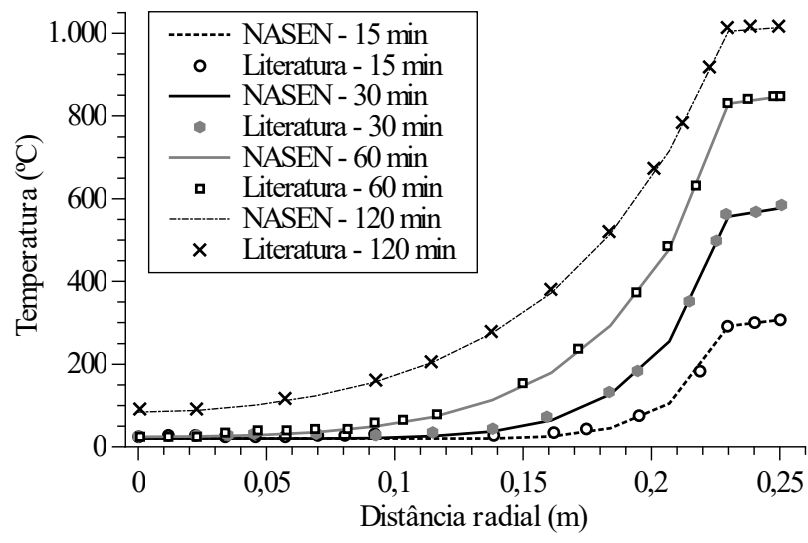

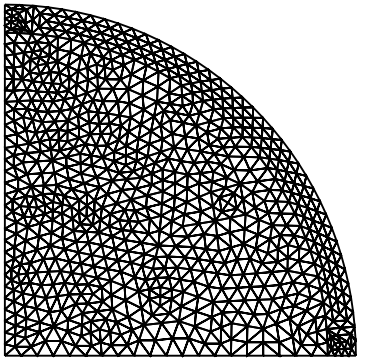

Malha de Elementos Finitos

Elemento Triangular $\#$ nox $=794 \quad$ \#elem $=1586$

Figura 4: Perfil radial de temperatura do tubo circula aço-concreto e malha numérica.

A partir de uma análise dos resultados é possível notar um bom ajuste do programa NASEN e os resultados da literatura de referência. Observa-se também que a temperatura no tubo de aço pouco varia - permanecendo quase constante, esse comportamento é por conta que o aço apresenta uma alta condutividade térmica. Em contrapartida, pela baixa condutividade do concreto, as 
temperaturas no centro do pilar são menores em comparação com as temperaturas medidas nas extremidades do tubo.

Por fim, o terceiro caso é uma seção transversal de uma coluna de concreto em forma de $\mathrm{T}$. Todas as faces da estrutura estão expostas ao incêndio padrão, conforme ilustra a Figura 2c. Diferentemente aos casos anteriores, conforme sugerido por Liu [8], adota-se uma emissividade de 0,8 e o coeficiente de convecção para as faces expostas ao incêndio igual a $12 \mathrm{~W} / \mathrm{m}^{2}{ }^{\circ} \mathrm{C}$.

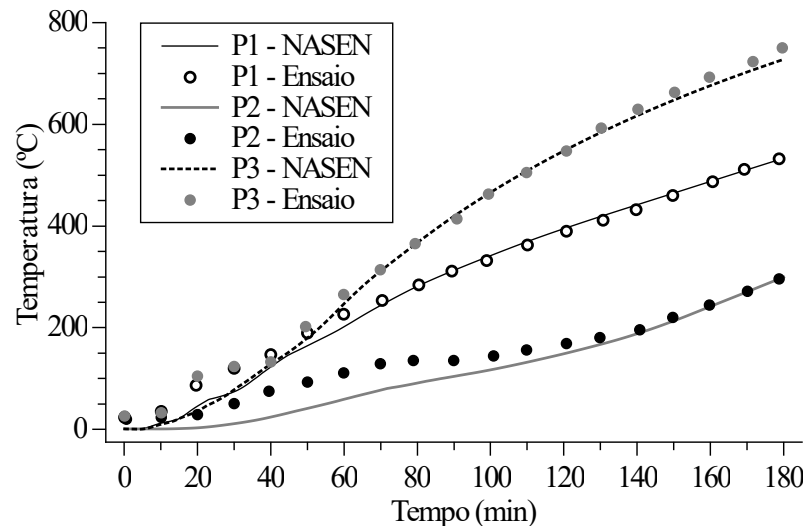

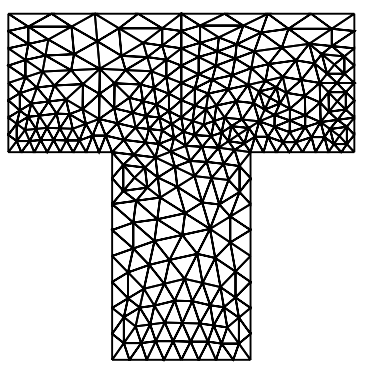

Malha de Elementos Finitos

Elemento Triangular $\#$ nox $=305 \quad$ \#elem $=608$

Figura 5: Evolução de temperatura no pilar de concreto em T e malha numérica.

A Figura 5 apresenta os resultados da variação de temperatura com o tempo para os pontos P1, P2 e P3 contido na coluna de concreto (ver Figura 2c), tendo como referência os dados experimentais obtidos em $\mathrm{Xu}$ e $\mathrm{Wu}$ [13]. A resposta obtida pelo programa computacional NASEN exibe uma boa performance em comparação aos testes experimentais. Quantitativamente, no tempo 180 min, tem-se um erro percentual de aproximadamente $0,008 \%, 0,85 \%$ e $3 \%$ para os pontos 1,2 e 3 , respectivamente.
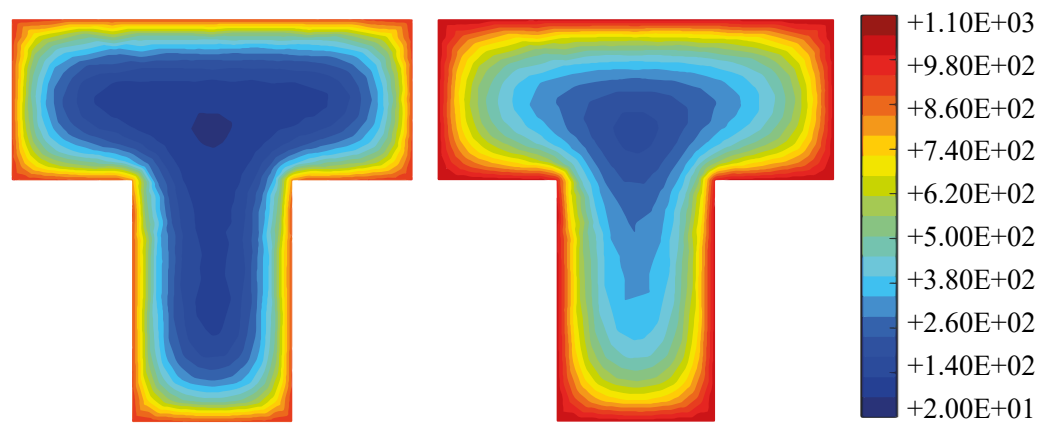

Figura 6: Campo de temperatura $\left({ }^{\circ} \mathrm{C}\right)$ para $60 \mathrm{~min}$ (esquerda) e $120 \mathrm{~min}$ (direita).

Em projetos e análises de estruturas em condição de incêndio, uma maneira usual de visualizar os resultados de uma análise térmica é por meio do campo bidimensional de temperatura. A Figura 6 exibe a distribuição de temperatura para $60 \mathrm{~min}$ e $120 \mathrm{~min}$ de exposição ao fogo. Essa forma de pós-processamento é interessante para avaliar as regiões críticas da seção transversal do elemento estrutural exposta aos elevados gradientes térmicos provocados pelo incêndio. 


\section{Conclusões}

O vigente trabalho teve como objetivo realizar uma avaliação de performance do módulo específico do programa NASEN. A análise térmica bidimensional em estruturas sob condição de incêndio é modelada pelo programa NASEN/TA-FIRE. Para efetuar a validação do módulo térmico foram realizados três casos testes, buscando, em cada caso, verificar o desempenho do programa diante os resultados obtidos por procedimentos normativos, simulações numéricas ou dados experimentais. O trabalho direcionou as análises para as estruturas envolvendo concreto.

Diante os resultados obtidos nos casos estudados pelo programa computacional desenvolvido, as respostas mostram-se coerentes com as soluções de referência, evidenciando o bom desempenho do módulo para a análise térmica bidimensional transiente não linear. Além disso, por meio dos exemplos explorados no trabalho foi possível verificar a versatilidade do programa em analisar diferentes geometrias, materiais e condições de contorno.

\section{Referências}

[1] ABNT NBR 14323. Projeto de estruturas de aço e de estruturas mistas de aço e concreto de edifícios em situação de incêndio. Rio de Janeiro, 2013.

[2] ABNT NBR 15200. Projeto de estruturas de concreto em situação de incêndio. Rio de Janeiro, 2012 .

[3] Bergman, T. L., Lavine, A. S., Incropera, F. P. \& Dewitt, D. P. Fundamentals of heat and mass transfer. New York: John Wiley \& Sons, 2011.

[4] EN 1992-1-2. Eurocode 2: Design of concrete structures-part 1-2: General rules-structural fire design. European Standards, London, CEN, Brussels, Belgium, 2004.

[5] EN 1993-1-2. Eurocode 3: Design of steel structures - part 1-2: General rules - structural fire design. European Standards, London, CEN, Brussels, Belgium, 2005.

[6] ISO 834. Fire resistance tests-elements of building construction. International Organization for Standardization, Geneva, Switzerland, Revision of frst edition ISO 834:1975, 1999.

[7] Landesmann, A. Modelo Não-Linear Inelástico para Análise de Estruturas Metálicas Aporticadas em Condições de Incêndio. Tese de Doutorado, COPPE/UFRJ, RJ, Brasil, 2003.

[8] Liu, Y. J. Modeling and programming of reinforced concrete structures exposed to fires. Ph.D. Dissertation, Department of Civil Engineering, Dalian Univeristy of Technology, Dalian, China (in Chinese), 2002.

[9] Neves, N. S.. Modelo computacional avançado para análise de estruturas sob ação de gradientes térmicos. Dissertação (Mestrado) — Universidade Federal do Espírito Santo, Vitória, 2019.

[10] Pierin, I., Silva, V., \& Rovere, H. L.. Thermal analysis of two-dimensional structures in fire. Revista IBRACON de Estruturas e Materiais, SciELO Brasil, v. 8, n. 1, p. 25-36, 2015.

[11] Pires, D., Barros, R. C., Rocha, P. A. S. \& Silveira, R. A. D. M.. Thermal analysis of steel-concrete composite cross sections via CS-ASA/FA. REM-International Engineering Journal, SciELO Brasil, v. 71, n. 2, p. 149-157, 2018.

[12] Reddy, J. N. \& Gartling, D. K.. The finite element method in heat transfer and fluid dynamics. New York: CRC press, 2010.

[13] Xu, Y.Y. \& Wu, B. Fire resistance of reinforced concrete columns with L-, T-, and+-shaped cross-sections. Fire Safety Journal, v. 44, n. 6, p. 869-880, 2009.

[14] Yin, J., Zha, X.-X. \& Li, L.-y. Fire resistance of axially loaded concrete filled steel tube columns. Journal of Constructional Steel Research, Elsevier, v. 62, n. 7, p. 723-729, 2006. 